Check for updates

Cite this: RSC Chem. Biol., 2020, 1,436

Received 15th July 2020,

Accepted 11th September 2020

DOI: 10.1039/d0cb00126k

rsc.li/rsc-chembio

\title{
Xanthine-based photoaffinity probes allow assessment of ligand engagement by TRPC5 channels $\nmid$
}

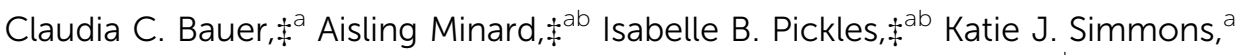 \\ Eulashini Chuntharpursat-Bon, ${ }^{a}$ Matthew P. Burnham, ${ }^{c}$ Nikil Kapur, ${ }^{d}$ \\ David J. Beech, ${ }^{a}$ Stephen P. Muench, (D) ef Megan H. Wright, (D) bf \\ Stuart L. Warriner ${ }^{\text {bf }}$ and Robin S. Bon (D) *af
}

\begin{abstract}
TRPC1/4/5 cation channels are emerging drug targets for the treatment of, amongst others, central nervous system (CNS) disorders, kidney disease, and cardiovascular and metabolic disease. Various small-molecule TRPC1/4/5 modulators have been reported, including highly potent xanthine derivatives that distinguish between specific TRPC1/4/5 tetramers. However, tools to profile ligand engagement by TRPC1/4/5 channels in live cells are lacking. Here, we report a set of potent xanthine-based photoaffinity probes that functionally mimic the xanthines Pico145 and AM237. Using these probes, we have developed a photoaffinity labelling protocol for TRPC 5 channels, providing the first method for the quantitative assessment of binding interactions of TRPC5 with small molecules in cells. This method could be important for drug discovery efforts targeting the xanthine/lipid binding site of TRPC1/4/5 channels.
\end{abstract}

\section{Introduction}

Transient Receptor Potential Canonical (TRPC) proteins are members of the TRP superfamily, which also includes TRPV, TRPA and TRPM..$^{1-8}$ Like other TRPs, TRPC proteins form tetrameric, non-selective cation channels permeable by $\mathrm{Na}^{+}$and $\mathrm{Ca}^{2+}$. Based on sequence homology, the human TRPC proteins are divided into two clusters: TRPC1/4/5 and TRPC $3 / 6 / 7,{ }^{9}$ while TRPC2 is encoded by a pseudogene in human. ${ }^{10}$ TRPC4 and TRPC5 are the most closely related TRPC proteins ${ }^{7}$ (70\% sequence identity; BLAST search $^{8}$ ). TRPC1/4/5 proteins can assemble to form homo- or heterotetrameric channels, each with their own biophysical characteristics (e.g., voltage dependence, ion selectivity), cellular

\footnotetext{
${ }^{a}$ Leeds Institute of Cardiovascular and Metabolic Medicine, LIGHT Laboratories, University of Leeds, Leeds LS2 9JT, UK. E-mail: r.bon@leeds.ac.uk

${ }^{b}$ School of Chemistry, University of Leeds, Woodhouse Lane, Leeds LS2 9JT, UK

${ }^{c}$ Discovery Sciences, R\&B Biopharmaceuticals, AstraZeneca, Alderley Park, UK

${ }^{d}$ School of Mechanical Engineering, University of Leeds, Woodhouse Lane, Leeds LS2 9JT, UK

${ }^{e}$ School of Biomedical Sciences, University of Leeds, Woodhouse Lane, Leeds LS2 9JT, UK

${ }^{f}$ Astbury Centre for Structural Molecular Biology, University of Leeds, Woodhouse Lane, Leeds LS2 9JT, UK

$\dagger$ Electronic supplementary information (ESI) available: Synthesis and characterisation of compounds, design of LED photocrosslinker setup, and additional data table and figures. See DOI: 10.1039/d0cb00126k

\$ These authors contributed equally.
}

function, and pharmacological profile. ${ }^{11}$ TRPC1 may not form functional homomeric channels, but is an important contributor to heteromeric channels, for example with TRPC4 and/or TRPC5. ${ }^{1,6,9-13}$ However, there remains a lack of insight into the exact compositions and stoichiometries of native TRPC1/4/5 channels, which may vary depending on, e.g., cell type, tissue and cellular state. The implication of TRPC1/4/5 in a wide range of physiological and pathological mechanisms has driven the search for potent and selective channel modulators for studies in cells, tissues and animal models. ${ }^{11,12}$ Because disruption of the Trpc4/5 genes $^{13}$ and global expression of a dominant-negative mutant TRPC $^{14}$ do not cause catastrophic phenotypes in rodents, TRPC1/4/5 inhibition may be particularly promising as a therapeutic strategy. Indeed, clinical trials with at least two TRPC1/4/5 inhibitors have been announced by Hydra Biosciences/Boehringer Ingelheim (the TRPC4/5 channel inhibitor BI 135889 for treatment of anxiety/CNS disorders) and Goldfinch Bio (the TRPC5 channel inhibitor GFB-887 for genetically-driven kidney disease). The structures of these clinical candidates have not been disclosed to date.

Chemically-diverse small-molecule TRPC1/4/5 modulators have been reported, but for most of these the modes-of-action are poorly understood..$^{2,11,12,15,16}$ Cellular targets may be diverse, and even direct binders may have different TRPC1/4/5 binding sites. The most potent, selective and efficacious TRPC1/4/5 activator is the natural product (-)-englerin A (EA). ${ }^{17-19}$ 
A<smiles>Cn1c(=O)n(CCCO)c(=O)c2c1nc(Oc1cccc(OC(F)(F)F)c1)n2Cc1ccc(Cl)cc1</smiles>

1; Pico145 (HC-608); inhibits TRPC1/4/5 channels<smiles>Cn1c(=O)n(CCCO)c(=O)c2c1nc(Oc1cccc(Cl)c1)n2Cc1ccc(Cl)cc1</smiles>

2; HC-070; inhibits TRPC1/4/5 channels

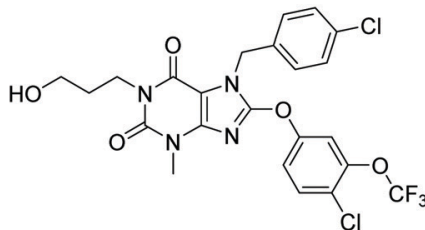

$\mathrm{Cl}$

3; AM237; activates TRPC5:C5; inhibits TRPC4:C4, TRPC1:C4 and TRPC1:C5 channels

B
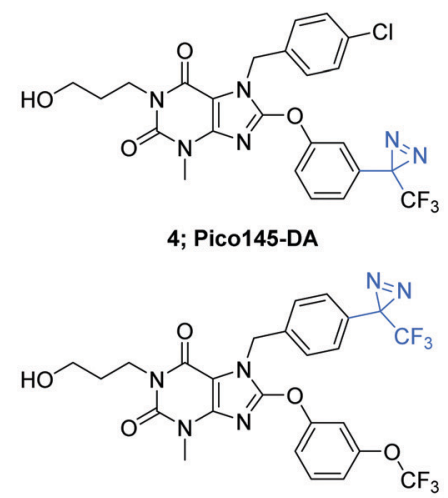

6; Pico145-DA2

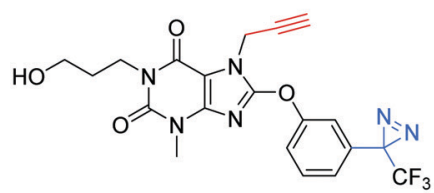

5; Pico145-DAAlk

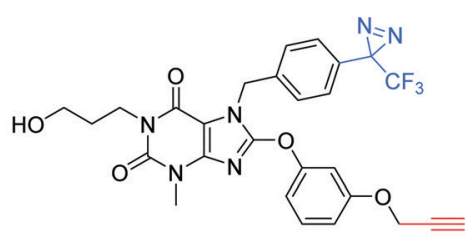

7; Pico145-DAAlk2

Fig. 1 Structures of xanthine derivatives discussed in this work. (A) Structures of previously described xanthine-based TRPC1/4/5 modulators Pico145 (HC-608) 1, HC-070 2 and AM237 3. (B) Structures of new xanthine-based photoaffinity probes described in this work: Pico145-DA 4 (Pico145-diazirine), Pico145-DAAlk 5 (Pico145-diazirine-alkyne), Pico145-DA2 6 (Pico145-diazirine-2) and Pico145-DAAlk2 7 (Pico145-diazirine-alkyne-2). Changes to the Pico145 structure are highlighted in blue (diazirine photocrosslinkers) and red (alkyne handles).

Although EA has been used in animals, ${ }^{20}$ its toxicity ${ }^{19,21}$ and instability in rodent plasma ${ }^{19}$ need to be carefully considered in study design. The most promising and well-characterised TRPC1/4/5 inhibitors are two closely-related xanthines, Pico145 1 (also called HC-608) and HC-070 2, which originally appeared in a patent assigned to Hydra Biosciences (Fig. 1). ${ }^{22}$ Pico145 is the most potent inhibitor of TRPC1/4/5 channels to date, ${ }^{23,24}$ with the highest potencies against heteromeric channels and high selectivity against 8 related ion channels. Just et al. reported the anxiolytic and antidepressant effects in mice of HC-070. ${ }^{25}$ As part of this study, activities of HC-070 and Pico145 were confirmed against human, mouse and rat TRPC1/4/5 channels. In addition, both compounds were shown to be $>400$-fold selective against $>100$ ion channels, receptors, enzymes, kinases and transporters ( $>2000$-fold for most) and to be orally bioavailable. Therefore, Pico145 and HC-070 are considered valuable chemical probes for functional studies of TRPC1/4/5 channels in cells and animals. ${ }^{21,25-27}$ In addition, we reported that AM237, a close analogue of Pico145 and HC-070, is an activator of homomeric TRPC5:C5 channels, but an inhibitor of TRPC4:C4, TRPC1:C5 and TRPC1:C4 channels. ${ }^{28}$

Pico145 is a competitive antagonist of both EA- and AM237mediated TRPC5:C5 activation. ${ }^{17,28}$ Pico145 also inhibits TRPC1/4/5 channels activated by carbachol ${ }^{25}$ and sphingosine-1phosphate (S1P) ${ }^{23}$ (which act via G-protein signalling), and by the direct agonist $\mathrm{Gd}^{3+}$, although low concentrations of Pico145 $(10 \mathrm{pM})$ can also potentiate $\mathrm{Gd}^{3+}$-induced currents mediated by TRPC $4 .^{23}$ These data suggest that xanthines such as Pico145 and AM237 occupy a well-defined, high-affinity binding site essential to TRPC1/4/5 channel gating to stabilise open or closed TRPC5 channel states, depending on subunit composition and xanthine substituent pattern. We recently reported cryo-EM structures of human TRPC5:C5 in complex with Pico145, revealing that Pico145 binds to a conserved lipid binding site of TRPC5, where it displaces a bound phospholipid. ${ }^{29}$ A subsequent report of a cryo-EM structure of TRPC5:C5 in complex with HC-070 ${ }^{30}$ suggested that HC-070 binds to the same site as Pico145, with a near-identical binding pose. These studies highlight targeting of the conserved lipid binding site of TRPC $1 / 4 / 5$ channels as a promising strategy for TRPC $1 / 4 / 5$ drug discovery.

An important step in drug discovery is the assessment of cellular target engagement and occupancy by candidate molecules. ${ }^{31-34}$ Photoaffinity labelling (PAL) ${ }^{35-37}$ can be used to identify, characterise and validate interactions of small molecules with membrane proteins and ion channels, ${ }^{38-42}$ which may readily lose their affinity for non-covalent ligands under various experimental conditions. PAL results in the formation of covalent bonds between a photoaffinity probe and its cellular target(s). The initiation of labelling with a specific wavelength of light enables spatio-temporal control and allows straight-forward control experiments in the absence of light.

The structure and pharmacological profile of a photoaffinity probe must closely resemble those of its parent compound, and the placement of the photoreactive group must favour intermolecular crosslinking over intramolecular rearrangement. ${ }^{40}$ 
A high degree of nonspecific labelling is not uncommon in PAL, requiring deconvolution of specific and non-specific labelling events. ${ }^{43,44}$ PAL on ion channels is particularly challenging: low expression levels, in combination with low photolabelling efficiencies common in PAL, often result in only small quantities of labelled protein. Diazirines are the smallest photoreactive groups suitable for PAL. Upon irradiation with UV light (365 $\mathrm{nm})$, they generate highly reactive carbene species that rapidly insert into proximal $\mathrm{C}-\mathrm{H}$ or heteroatom-H bonds. ${ }^{37,45}$ Incorporation of diazirines allows the development of photoaffinity probes through minimal structural variation of small molecules. $^{46-48}$

We hypothesised that PAL would be a suitable approach to assess cellular target engagement of TRPC1/4/5 modulators. Here, we report the development of a PAL protocol for TRPC5 channels. Modification of Pico145 with diazirines and alkyne handles gave a set of photoaffinity probes that functionally mimic either Pico145 or AM237. Our 2-step labelling protocol based on PAL and copper-catalysed azide-alkyne cycloadditions $(\mathrm{CuAAC})^{49}$ provided the first proof of a direct interaction between xanthines and TRPC5 in live cells, and allowed the quantitative assessment of TRPC5 binding by Pico145 in human cells. This technology may allow assessment of target engagement of other TRPC1/4/5 modulators and elucidation of their mode-of-action, and thereby underpin TRPC1/4/5 drug discovery targeting the xanthine/lipid binding site.

\section{Results}

\section{Development of xanthine-based photoaffinity probes}

Analysis of semi-quantitative TRPC5 assay data for 621 compounds described in the patent literature ${ }^{22}$ revealed the $N-7$ and $C-8$ substituents of Pico145 to be the most suitable sites for incorporation of functional handles (Fig. S1, ESI $\dagger$ ). Aiming for maximal structural similarity to Pico145 itself, we designed and synthesised a set of four Pico145 photoaffinity probes 4-7 (Fig. 1B), all containing the (trifluoromethyl)(phenyl)diazirine motif commonly used in diazirine photocrosslinkers. ${ }^{37,50}$ Pico145diazirine (Pico145-DA; 4) and Pico145-diazirine-2 (Pico145-DA2; 6) are structurally most similar to Pico145 and were made to test the functional effect of incorporation of a diazirine group. The compounds designed for our photocrosslinking studies, Pico145-diazirine-alkyne (Pico145-DAAlk; 5) and Pico145-diazirinealkyne-2 (Pico145-DAAlk2; 7) incorporate an additional alkyne handle that allows bioorthogonal labelling with various reporter groups, such as biotin or a fluorophore, through CuAAC. The compounds were synthesised, purified to homogeneity, and chemically characterised as described in the ESI, $\dagger$ Methods.

\section{Xanthine-based photoaffinity probes are nanomolar TRPC1/4/5 modulators}

The effects of xanthine photoaffinity probes on TRPC1/4/5 ion channel activity were assessed by intracellular calcium $\left(\left[\mathrm{Ca}^{2+}\right]_{\mathrm{i}}\right)$ measurements in HEK T-REx cells expressing tetracyclineinducible TRPC5-SYFP2, TRPC4-SYFP2, TRPC5-C1 or TRPC4-C1, using Fura-2 as the calcium indicator (see Methods). To test TRPC1/4/5 channel inhibition, cells were pre-treated with increasing concentrations of photoaffinity probes 4-7 before starting $\left[\mathrm{Ca}^{2+}\right]_{\mathrm{i}}$ monitoring, followed by application of the TRPC1/4/5 channel activator (-)-englerin A (EA). Because initial experiments with TRPC5-expressing cells revealed concentrationdependent increases in baseline fluorescence upon pre-incubation with 4 and 5 (reminiscent of $\mathrm{AM} 237^{28}$ ) these compounds were further evaluated in activation mode, in which acute effects of application of 4 and 5 on $\left[\mathrm{Ca}^{2+}\right]_{\mathrm{i}}$ were monitored. The results of these experiments are depicted in Fig. 2 and ESI, $\dagger$ Fig. S2-S5. All four photoaffinity probes 4-7 modulated TRPC1/4/5 activities in a concentration-dependent manner, with half-maximal effects at concentrations in the nanomolar range. Unlike Pico145, and similar to AM237, Pico145-DAAlk 5 activated TRPC5:C5 channels $\left(\mathrm{EC}_{50} 19 \mathrm{nM}\right.$; Fig. 2A and B), and inhibited other TRPC1/4/5 channels ( $\mathrm{IC}_{50}$ values 46-196 nM; ESI, $\dagger$ Fig. S2). The activation of TRPC5:C5 by Pico145-DAAlk 5 (100 nM) could be inhibited by Pico145 in a concentration-dependent manner (IC $501.8 \mathrm{nM}$; Fig. 2C and D). Pico145-DA 4 also activated TRPC5:C5 (EC 50 $13 \mathrm{nM}$; ESI, $\dagger$ Fig. S3A and B) and inhibited other TRPC1/4/5 channels ( $\mathrm{IC}_{50}$ values $1.4-40 \mathrm{nM}$; ESI, $\dagger$ Fig. S3C-H), suggesting that the activating effect on TRPC5:C5 is a consequence of incorporation of the diazirine group into the $C$-8 substituent rather than the alkyne handle on $N$-7. In contrast, Pico145DAAlk2 7 inhibited TRPC5:C5 ( $\mathrm{IC}_{50} 26 \mathrm{nM}$; Fig. 2E and F) as well as other TRPC1/4/5 channels ( IC $_{50}$ values 2.0-17 nM; ESI, $\dagger$ Fig. S4), and similar effects were seen for Pico145-DA2 6 $\left(\mathrm{IC}_{50}\right.$ values 1.3-18 $\mathrm{nM}$; ESI, $\dagger$ Fig. S5). A comparison of the $\mathrm{EC}_{50}$ and $\mathrm{IC}_{50}$ values, shown in Table 1, suggests that - in the context of TRPC1/4/5 channels - Pico145-DA 4 and Pico145DAAlk 5 mimic AM237, while Pico145-DA2 6 and Pico145DAAlk2 7 mimic Pico145. In addition, the four photoaffinity probes retained high potency against TRPC1/4/5 channels compared to AM237 and Pico145. Note that Pico145 potency at TRPC1/4/5 channels can be reduced by higher EA concentrations, suggesting competition of the compounds. Therefore, the different concentrations of EA used as activator in inhibition assays with TRPC4:C4, TRPC4-C1 and TRPC5-C1 may exaggerate differences in $\mathrm{IC}_{50}$ values between compounds.

Docking of compounds 4-7 to the Pico145/lipid binding site of TRPC5: $5^{29}$ revealed potential binding modes. Similar to the best predictions for the binding mode of Pico145, ${ }^{29}$ these simulations suggest that the xanthine cores of all four compounds 4-7 make $\pi$-stacking interactions with F576, while their 3-hydroxypropyl groups make hydrogen bonding interactions with Q573 and W577 (Fig. 3). The top-scoring poses of Pico145DA2 6 and Pico145-DAAlk2 7 (Fig. 3C and D) were near-identical to that of Pico145 in the cryo-EM structure, ${ }^{29}$ with their respective $\mathrm{OCF}_{3}$ and $O$-propargyl groups oriented towards the hydrophobic tunnel made by L528 and V579. In addition, the diazirine groups at the para-position of the $N-7$ benzyl substituents of 6 and 7 were easily accommodated in the binding pocket. In contrast, the top-scoring poses for Pico145-DA 4 and Pico145-DAAlk 5 suggest that a slight twist of their $C-8$ substituents is required to avoid potential clashes between 
A

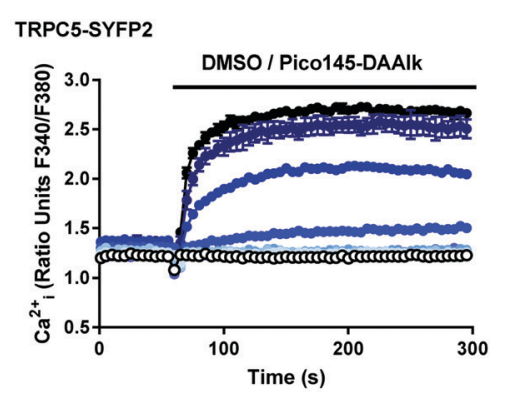

C

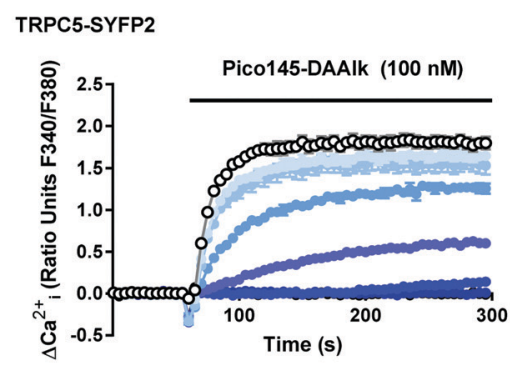

E

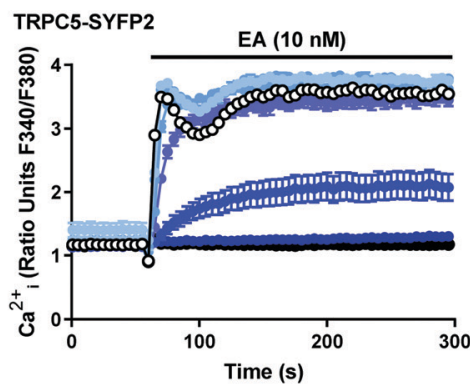

B

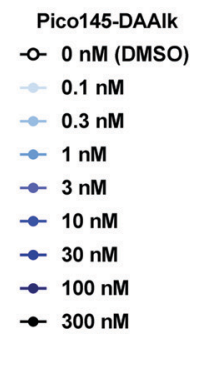

TRPC5-SYFP2

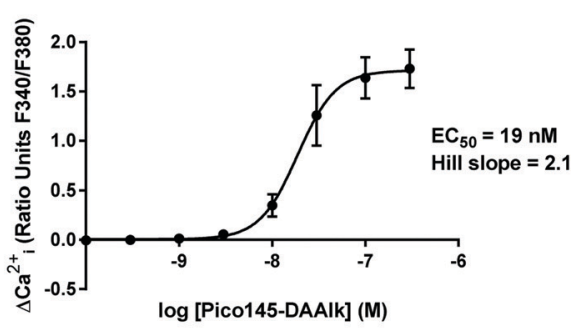

D

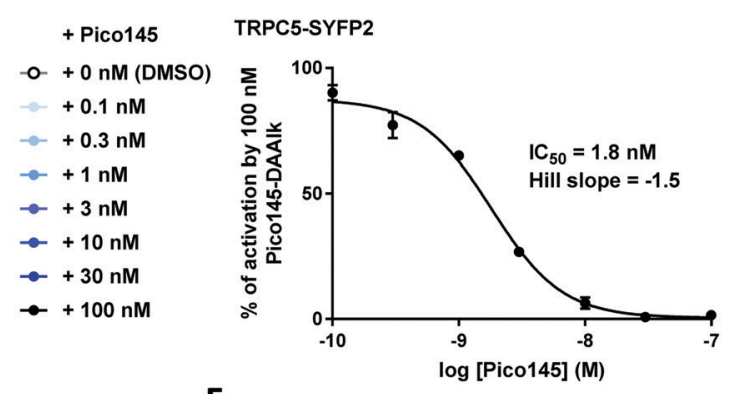

F

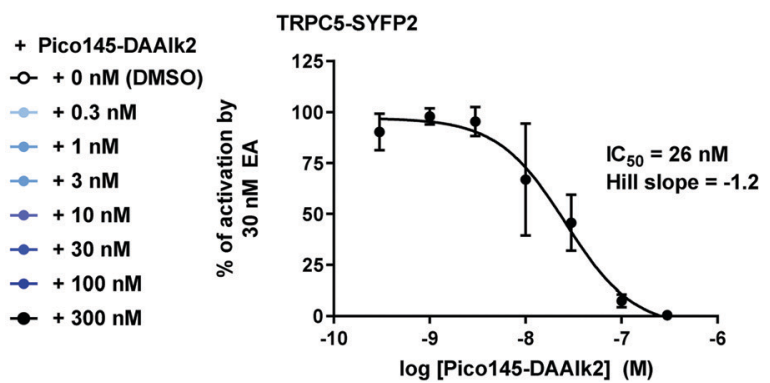

Fig. 2 Pico145-DAAlk 5 and Pico145-DAAlk2 7 are potent TRPC5:C5 channel modulators. (A) Representative traces from a single 96-well plate ( $N=6$ ) showing an increase in $\left[\mathrm{Ca}^{2+}\right]_{i}$ in response to $0.1-300 \mathrm{nM}$ Pico145-DAAlk 5 in (Tet+) HEK T-REx cells overexpressing hTRPC5-SYFP2. (B) Concentrationresponse data for experiments in (A), showing mean responses \pm SEM ( $n / N=3 / 18)$. Responses were calculated at $150-200 \mathrm{~s}$, compared to baseline at $0-55 \mathrm{~s}$. (C) Representative traces from a single 96 -well plate $(N=6)$ showing an inhibition by $0.1-100 \mathrm{nM}$ Pico145 1 of the increase in [Ca $\left.{ }^{2+}\right]_{i}$ in response to 100 nM Pico145-DAAlk 5 in (Tet+) HEK T-REx cells overexpressing hTRPC5-SYFP2. (D) Concentration-response data for experiments in (C) showing mean responses \pm SEM $(n / N=3 / 18)$. Responses were calculated at 150-200 s, compared to baseline at 0-55 s. (E) Representative traces from a single 96-well plate $(N=6)$ showing an inhibition by $0.3-300 \mathrm{nM}$ Pico145-DAAlk 7 of the increase in $\left[\mathrm{Ca}^{2+}\right]_{\mathrm{i}}$ in response to $10 \mathrm{nM}$ EA in (Tet+) HEK T-REx cells overexpressing hTRPC5-SYFP2. (F) Concentration-response data for experiments in (E), showing mean responses \pm SEM ( $n / N=3 / 18$ ). Responses were calculated at 250-295 s, compared to baseline at 0-55 s.

their (trifluoromethyl)diazirine groups and nearby residues V610, V579 and L521 (Fig. 3A, B and ESI, † Fig. S6). These simulations are consistent with the hypothesis that AM237 would also need to rearrange its $C-8$ aryl substituent to avoid a clash with $\mathrm{L} 521,{ }^{29}$ and suggest that changes to the $C-8$ aryl substitution pattern may be a valid strategy for the rational development of xanthine-based TRPC5:C5 activators. However, further structural insight into xanthine-based TRPC5:C5 activation is required, for example through the determination of open channel structures, which have not been reported to date.

The high similarity of compounds 4-7 to Pico145 and/or AM237 (in terms of structure, functional effect and potency as TRPC1/4/5 modulators, and predicted binding modes) in combination with their increased predicted aqueous solubility (especially in case of Pico145-DAAlk 5; ESI, $\dagger$ Table S1) are consistent with properties of well-designed xanthine-based photoaffinity probes for cellular ligand engagement studies.

\section{Pico145-DAAlk and Pico145-DAAlk 2 allow photoaffinity labelling of TRPC5 in human cells}

To investigate the potential of xanthine-based photoaffinity probes for TRPC ligand engagement studies, we set out to develop a photoaffinity labelling (PAL) protocol, making use of the alkyne handles of the diazirine-alkyne probes Pico145-DAAlk 5 and Pico145-DAAlk2 7 (Fig. 4). In an initial experiment, $300 \mathrm{nM}$ Pico145-DAAlk was used to photolabel and - after CuAAC with TAMRA azide - detect modified proteins by fluorescent gel scanning (ESI, $\dagger$ Fig. S7A). Labelled proteins were only detected 
Table 1 Summarised $\mathrm{EC}_{50}$ and $\mathrm{IC}_{50}$ values for Pico145 1, AM237 3, and photoaffinity probes $4-7$ against TRPC1/4/5 channels ${ }^{a}$

\begin{tabular}{|c|c|c|c|c|c|}
\hline Compound & TRPC5:C5 $\mathrm{EC}_{50}(\mathrm{nM})$ & TRPC5:C5 IC $_{50}(\mathrm{nM})$ & TRPC4:C4 IC 50 (nM) & TRPC4-C1 IC $50(\mathrm{nM})$ & TRPC5-C1 IC $_{50}(\mathrm{nM})$ \\
\hline $\operatorname{AM} 2373^{28}$ & 15 & & $7^{c}$ & $3^{d}$ & $4^{c}$ \\
\hline Pico145-DA 4 & 13 & & $40^{c}$ & $1.4^{c}$ & $4.9^{c}$ \\
\hline Pico145-DAAlk 5 & 19 & & $45^{c}$ & $121^{d}$ & $196^{c}$ \\
\hline Pico145-DA2 6 & & $18^{b}$ & $16^{c}$ & $1.3^{c}$ & $6.1^{c}$ \\
\hline Pico145-DAAlk2 7 & & $26^{b}$ & $17^{c}$ & $2.0^{c}$ & $6.2^{c}$ \\
\hline
\end{tabular}

${ }^{a}$ EA is a negative modulator of Pico145 potency, suggesting that Pico145 is a competitive inhibitor of EA. ${ }^{23}$ Therefore, lower EA concentrations used to activate TRPC1/4/5 channels may result in lower IC $_{50}$ values. ${ }^{b} 10 \mathrm{nM}$ EA was used as activator. ${ }^{c} 30 \mathrm{nM}$ EA was used as activator. ${ }^{d} 100 \mathrm{nM}$ EA was used as activator.

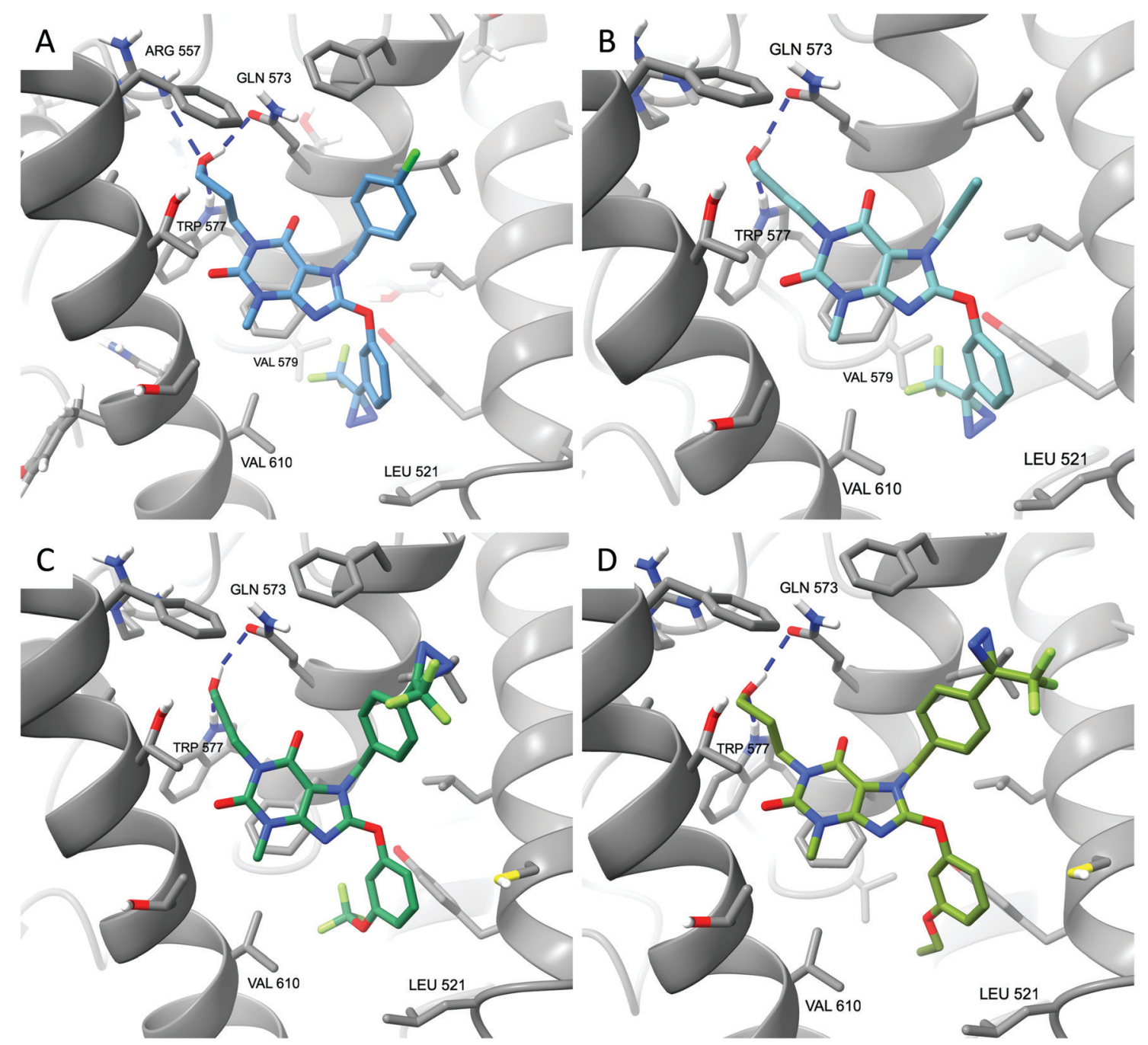

Fig. 3 Xanthine-based photoaffinity probes 4-7 have similar predicted binding modes in TRPC5:C5. (A) Top-scoring docked pose of Pico145-DA 4. (B) Top-scoring docked pose of Pico145-DAAlk 5. (C) Top-scoring docked pose of Pico145-DA2 6. (D) Top-scoring docked pose of Pico145-DAAlk2 7. All compounds were docked into a $36 \times 36 \times 36 \AA^{3}$ grid around the Pico145 binding site of TRPC5:C5 (PDB: 6 YSN).

in samples that had been subjected to both Pico145-DAAlk 5 and UV irradiation (ESI, $\dagger$ Fig. S7B and C), and included a protein of $\sim 140 \mathrm{kDa}$ present only in cells that express TRPC5-SYFP2 (ESI, $\dagger$ Fig. S7C and D). This is consistent with direct binding of Pico145DAAlk 5 to TRPC5-SYFP2. However, the aim of this work was to quantitatively probe interactions between xanthines and TRPC5, and the faint TRPC5-SYFP2 band was difficult to detect against the background of other labelled proteins. Therefore, we modified the photoaffinity labelling protocol to optimise reliable detection of TRPC5-xanthine interactions (see Methods).

HEK T-REx cells expressing TRPC5-SYFP2 were treated with Pico145-DAAlk 5 (300 nM), and subjected to irradiation with UV 


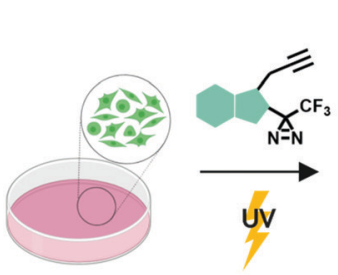

A

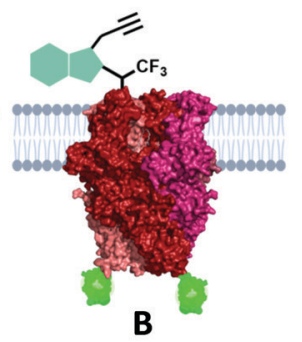

B
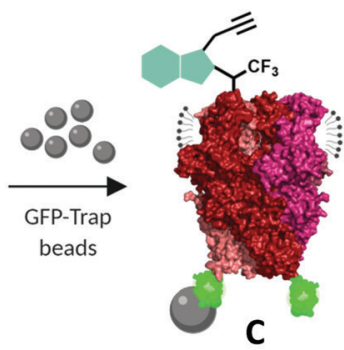
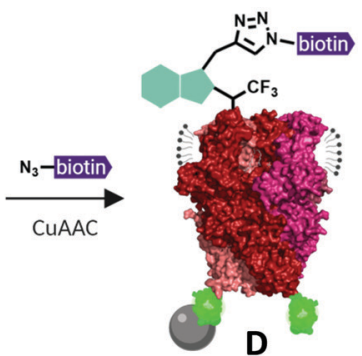

D

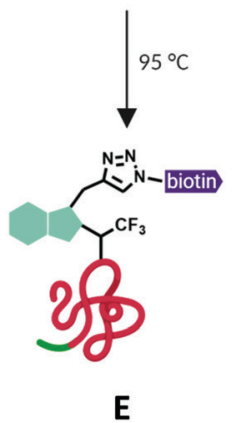

Fig. 4 Schematic representation of photoaffinity labelling protocol with Pico145-DAAlk 5 or Pico145-DAAlk2 7. HEK T-REx cells expressing TRPC5SYFP2 (A) were incubated with photoaffinity probes and irradiated with UV light (B). Following lysis and GFP-Trap immunoprecipitation (C), photolabelled TRPC5-SYFP2 was conjugated to TAMRA biotin azide (D) and eluted from GFP-Trap beads (E). Samples were separated using SDS-PAGE and analysed by western blotting ( $\mathrm{F}$ and $\mathrm{G}$ ). TRPC5 representation is based on its cryo-EM structure (PDB: $6 \mathrm{AEI}){ }^{51}$

light (365 nm; Analytik Jena AG photocrosslinker) for 25 minutes. Cells were then washed and lysed, before immunoprecipitation of TRPC5-SYFP2 using GFP-Trap affinity beads. An on-bead CuAAC reaction with TAMRA biotin azide (ESI, $\dagger$ Fig. S35) was then performed, after which proteins were eluted from the GFP-Trap beads, separated by SDS-PAGE and analysed by western blotting with Streptavidin-HRP to detect biotin-labelled proteins (Fig. 5A-(i)) and - after membrane stripping - anti-GFP to detect TRPC5-SYFP2 (Fig. 5A-(ii)). Input samples were also probed with anti-GFP to confirm successful TRPC5-SYFP2 expression (Fig. 5A-(iii)).
A

A

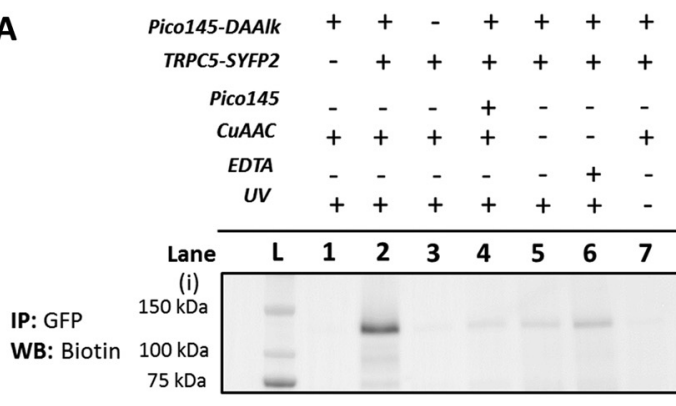

(ii)

IP: GFP

WB: GFP
B

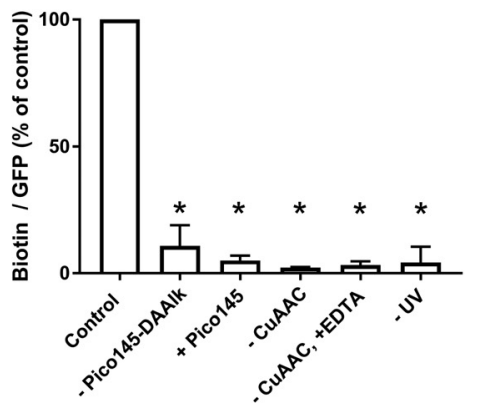

Input WB: GFP

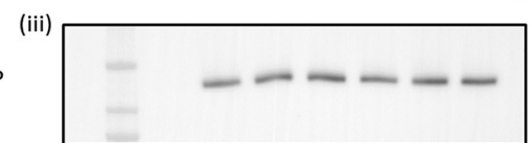

Fig. 5 Photoaffinity labelling of TRPC5 with Pico145-DAAlk 5. (A) Representative western blot after experiment depicted in Fig. 3. Samples were blotted with Streptavidin-HRP (i) and anti-GFP (ii; loading control). Western blot with anti-GFP of input (pre-GFP trap) samples was performed to confirm successful GFP-SYFP2 expression in HEK T-REx cells (iii). The data show that photolabelling of TRPC5-SYFP2 in HEK T-REx cells (lane 2) is dependent on expression of TRPC5-SYFP2 (lane 1), treatment with Pico145-DAAlk 5 (300 nM, 30 min, $37{ }^{\circ} \mathrm{C}$; lane 3), UV irradiation (365 nm, Analytik Jena AG photocrosslinker, $25 \mathrm{~min}$; lane 7), and CUAAC (TAMRA biotin azide $100 \mu \mathrm{M}, \mathrm{CuSO}_{4} 1 \mathrm{mM}, \mathrm{TCEP}_{1} \mathrm{mM}, \mathrm{TBTA} 800 \mu \mathrm{M} ; 1 \mathrm{~h}, 37^{\circ} \mathrm{C}$; lanes 5 and 6). In addition, Pico145 $(10 \mu \mathrm{M})$ inhibits photoaffinity labelling with Pico145-DAAlk 5 (lane 4). (B) Densitometry analysis of data presented in (A). $p<0.05$, all compared to control (+TRPC5-SYFP2, +Pico145-DAAlk probe; lane 2; one-way ANOVA with Dunnett's post-test). For '-CuAAC' experiments, CuSO 4 , TCEP and TBTA were replaced by their corresponding vehicles (water or DMSO) during the CUAAC step of the protocol. Lane $\mathrm{L}$ indicates protein molecular weight ladder. Pictures of complete western blots are shown in ESI, † Fig. S10. 
This analysis allowed identification (Fig. 5A) and quantification (Fig. 5B) of TRPC5 photolabelling products. The experiments revealed a band at $c a .140 \mathrm{kDa}$, corresponding to the expected molecular weight of TRPC5-SYFP2 (Fig. 5A, lane 2), which was absent when TRPC5-SYFP2 expression was not induced with tetracycline (Fig. 5A, lane 1). Control experiments demonstrated that Pico145-DAAlk (Fig. 4A, lane 3), UV irradiation (Fig. 5A, lane 7) and the CuAAC reagents (Fig. 5A, lanes 5 and 6) were all essential for detection of photolabelled TRPC5. In addition, in a competition PAL experiment, pre-treatment of cells with Pico145 $(10 \mu \mathrm{M})$ led to inhibition of TRPC5 labelling (Fig. 5A, lane 4), suggesting that PAL is mediated by a specific molecular interaction between TRPC5 and Pico145-DAAlk 5. Experiments with Pico145-DAAlk2 7 instead of Pico145-DAAlk 5, which were performed using a custom-built LEDbased photocrosslinker (see ESI, $\dagger$ Methods for details) that allowed reduction of irradiation time to 1 minute, revealed similar results (ESI, $\dagger$ Fig. S8 and S9). These results suggest that xanthine-based photoaffinity probes $\mathbf{5}$ and 7 can be used for ligand-directed PAL of TRPC5 protein in human cells, and (through competition assays) for quantitative assessment of target engagement of TRPC5 modulators (see below).

\section{Dependence of TRPC5 photolabelling on photoaffinity probe concentration}

To investigate the dependence of TRPC5 labelling on photoaffinity probe concentration, the quantitative PAL experiments were performed with either $0.3-300 \mathrm{nM}$ Pico145-DAAlk 5 or 0-1000 nM Pico145-DAAlk2 7. Western blots with StreptavidinHRP showed concentration-dependent detection of labelled TRPC5 (Fig. 6A, (i)), while reprobing of membranes with antiGFP, and blotting of input samples, showed consistent pulldown and expression of TRPC5-SYFP2 (Fig. 6A, (ii) and (iii)). Densitometry analysis showed that TRPC5 labelling increased with increasing concentrations of Pico145-DAAlk 5 (Fig. 6A and B) or Pico145-DAAlk2 7 (Fig. 6C and D). Although it would be tempting to fit dose-response curves to these data, it is possible that at higher concentration of photoaffinity probes, nonspecific photoaffinity labelling - of other sites than the modulatory binding site(s) - contributes to signal intensity. Because clear signals could be detected at $300 \mathrm{nM}$ of either Pico145DAAlk 5 or Pico145-DAAlk2 7, this concentration was chosen for further experiments.

\section{Assessment of Pico145 binding to TRPC5 in human cells by photoaffinity labelling}

The results described above suggest that our quantitative PAL protocol with Pico145-DAAlk 5 and Pico145-DAAlk2 7, when performed in competition mode, could potentially provide an indirect, quantitative method to assess binding of small molecules to TRPC5 in cells. To test this, HEK T-REx cells expressing TRPC5-SYFP2 were pre-treated with either DMSO or 0-3000 nM Pico145 1, before performing PAL experiments with
A

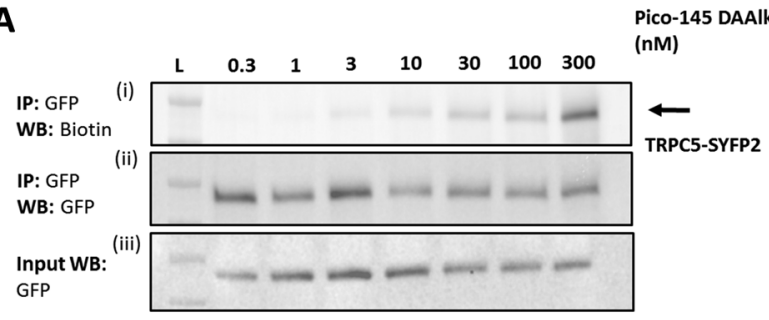

C
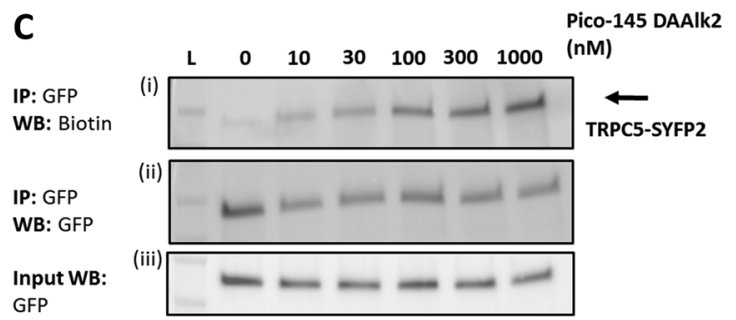

B

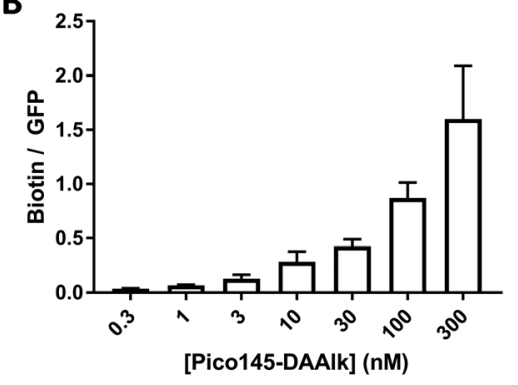

D

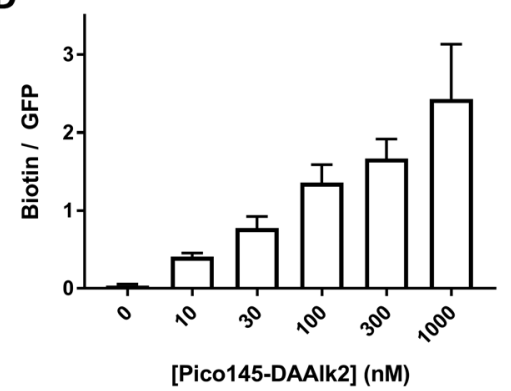

Fig. 6 Dependence of TRPC5 photoaffinity labelling on photoaffinity probe concentration. (A and C) Representative western blots showing concentration-dependent TRPC5 photolabelling with Pico145-DAAlk 5 (A) and Pico145-DAAlk2 7 (C). HEK T-REx cells overexpressing TRPC5-SYFP2 were treated with 0.3-300 nM Pico145-DAAlk 5 (A) or 0-1000 nM Pico145-DAAlk2 7 (C) for $30 \mathrm{~min}$ at $37^{\circ} \mathrm{C}$ and irradiated (365 nm). Following lysis and GFP pull-down, photolabelled TRPC5-SYFP2 was conjugated to TAMRA biotin azide (100 $\mu$ M) using CuAAC (CuSO $41 \mathrm{mM}$, TCEP 1 mM, TBTA $800 \mu$; $1 \mathrm{~h}$, $37{ }^{\circ} \mathrm{C}$ ). Samples were separated by SDS-PAGE and blotted with Streptavidin-HRP (i) and anti-GFP (ii; loading control). Western blot with anti-GFP of input (pre-GFP trap) samples was performed to confirm successful GFP-SYFP2 expression in HEK T-REx cells (iii). (B and D) Densitometry analysis of data presented in (A) and (C) respectively, showing mean data \pm SEM ( $n=3$ independent experiments each). Lane $L$ indicates protein molecular weight ladder. Pictures of complete western blots are shown in ESI, † Fig. S11. 
A

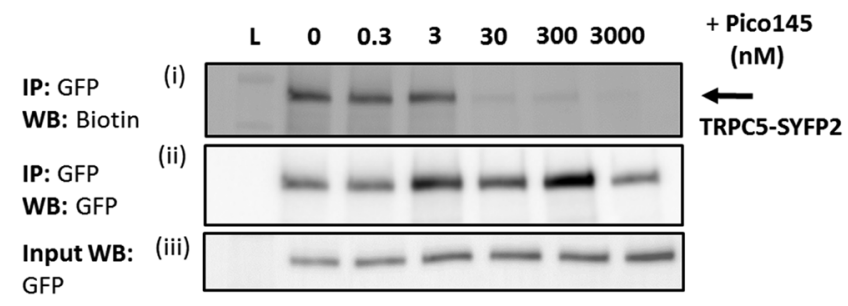

C

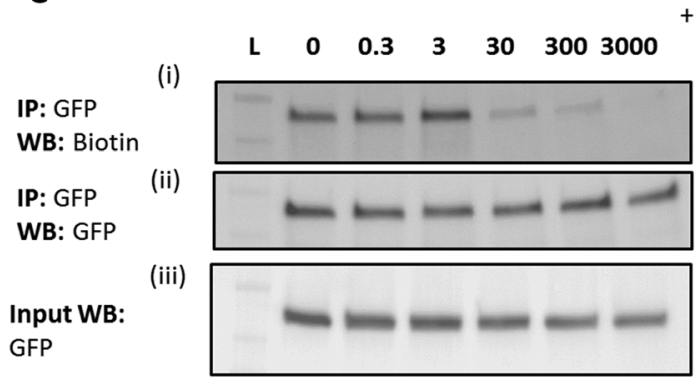

B

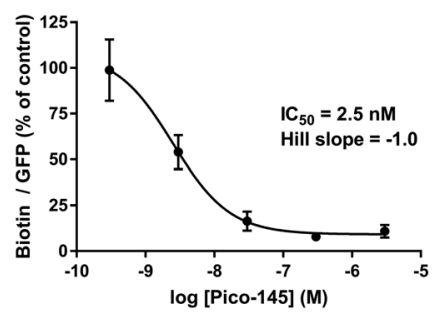

D

+ Pico145

(nM) TRPC5-SYFP2

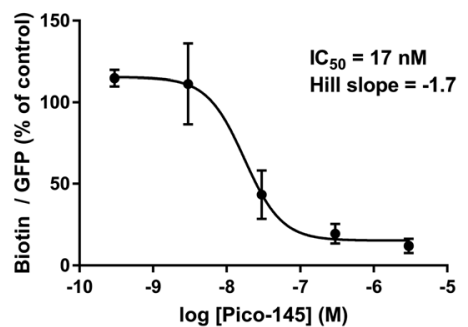

Fig. 7 Pico145 concentration-dependently inhibits TRPC5 photoaffinity labelling. (A and C) Representative western blots showing concentrationdependent inhibition by Pico145 of TRPC5 photolabelling with Pico145-DAAlk 5 (A) or Pico145-DAAlk2 7 (C). HEK T-REx cells overexpressing TRPC5SYFP2 were pre-treated with 0-3000 nM Pico145 (30 min, $37^{\circ} \mathrm{C}$ ) before being treated with $300 \mathrm{nM}$ Pico145-DAAlk 5 (A) or $300 \mathrm{nM}$ Pico145-DAAlk2 7 (C) for $30 \mathrm{~min}$ at $37{ }^{\circ} \mathrm{C}$ and irradiated $(365 \mathrm{~nm})$. Following lysis and GFP pull-down, TRPC5-SYFP2 was conjugated to TAMRA biotin azide (100 $\mu$ M) using CUAAC (CUSO 1 mM, TCEP $1 \mathrm{mM}$, TBTA $800 \mu \mathrm{M} ; 1 \mathrm{~h}, 37^{\circ} \mathrm{C}$ ). Samples were separated by SDS-PAGE and blotted with Streptavidin-HRP (i) and anti-GFP (ii; loading control). Western blot with anti-GFP of input (pre-GFP trap) samples was performed to confirm successful GFP-SYFP2 expression in HEK T-REx cells (iii). ( $B$ and D) Concentration-response data for experiments (A) and (C) respectively, showing mean data \pm SEM ( $n=3$ independent experiments each). Biotin/GFP was compared to control (0 nM Pico145). Lane $L$ indicates protein molecular weight ladder. Pictures of complete western blots are shown in ESI, † Fig. S12.

Pico145-DAAlk 5 (300 nM) or Pico145-DAAlk2 7 (300 nM). TRPC5-SYFP2 pull-down and expression was consistent across samples (Fig. 7A and C), and Pico145 concentration-dependently inhibited TRPC5 labelling by Pico145-DAAlk 5 ( IC $_{50} 2.5$ nM; Fig. 7A and $\mathrm{B}$ ) and Pico145-DAAlk2 7 ( $\mathrm{IC}_{50} 17 \mathrm{nM}$; Fig. 7C and D). Interestingly, the $\mathrm{IC}_{50}$ value of Pico145 inhibition of $\mathrm{PAL}$ with Pico145-DAAlk 5 (2.5 nM; Fig. 7A and B) is almost identical to the $\mathrm{IC}_{50}$ value of Pico145 inhibition of Pico145-DAAlk 5 evoked calcium entry (1.8 nM; Fig. 2C and D), even when taking into account the 3-fold difference in the concentration of Pico145-DAAlk 5 used in the respective assays (100 nM in calcium recording; $300 \mathrm{nM}$ in PAL). These data suggest that the functional activity of xanthine-based TRPC5 modulators such as Pico145 on TRPC5:C5 channels is directly related to a specific binding event between xanthines and TRPC5 protein.

\section{Discussion}

Through minimal structural variation of the most potent TRPC1/4/5 inhibitor, Pico145, we have developed a set of potent xanthine-based photoaffinity probes. Pico145-DA 4 and Pico145DAAlk 5 functionally mimic AM237; they activate TRPC5:C5 channels but inhibit TRPC4:C4, TRPC5-C1 and TRPC4-C1 channels. In contrast, Pico145-DA2 6 and Pico145-DAAlk2 7 functionally mimic Pico145; they inhibit all TRPC1/4/5 channels tested. We used Pico145-DAAlk 5 and Pico145-DAAlk2 7 to develop a new PAL/CuAAC protocol to measure cellular interactions between xanthines and TRPC5 protein. PAL of TRPC5 was successful within the same concentration range as the effects on TRPC5-mediated calcium influx, providing further evidence that xanthines modulate TRPC5 channels through direct interaction with TRPC5 protein, consistent with their behaviour in excised outside-out patch recordings ${ }^{23,28}$ and with our cryo-EM and site-directed mutagenesis studies. ${ }^{29}$ To the best of our knowledge, only one previous example of PAL on a TRPC channel had been reported: Kiyonaka et al. used a pyrazole derivative (Pyr-PP) incorporating a diazirine photocrosslinker and a ketone handle (for oxime ligation with a biotin hydroxylamine probe) to suggest that the TRPC3 channel inhibitor Pyr3 directly interacts with TRPC3 protein. $^{39}$

Competition PAL experiments with Pico145 allowed the indirect, quantitative assessment of the relative affinities of Pico145 and photoaffinity probes. The $\mathrm{IC}_{50}$ values determined for Pico145 in calcium recordings (Fig. 2C and D) and in competition PAL experiments (Fig. 7A and B) with Pico145DAAlk 5 are nearly identical. These results - in combination with the close structural similarities between Pico145 and Pico145-DAAlk 5 - are consistent with the hypothesis that xanthine-based TRPC5:C5 activators (e.g., AM237, Pico145-DA 4, and Pico145-DAAlk 5) and xanthine-based TRPC5:C5 inhibitors (e.g., Pico145) bind to identical site(s) of TRPC1/4/5 channels, 
leading to stabilisation of open or closed TRPC1/4/5 channel conformations. In addition, our docking studies suggest that, similar to AM237, ${ }^{29}$ Pico145-DA 4 and Pico145-DAAlk 5 contain structural features associated with TRPC5:C5 activation rather than inhibition (Fig. 3 and ESI, $\dagger$ Fig. S6). Despite extensive experimentation resulting in successful mass spectrometrybased peptide mapping of TRPC5 constructs (up to 99\% sequence coverage; not shown), we have not yet been able to determine which peptides/residues are labelled by xanthinebased photoaffinity probes. We hypothesise that this is the result of low efficiency of photolabelling of TRPC5, for which diazirines are notorious. ${ }^{37,52}$ Our cryo-EM studies revealed that up to four molecules of Pico145 can bind to four lipid binding sites of a TRPC5 tetramer. ${ }^{29}$ Although it is not yet known how many binding sites need to be occupied for channel modulation, xanthine-based TRPC1/4/5 modulators often display Hill slopes $>1$ in intracellular calcium measurements (Fig. 2 and ESI, $\dagger$ Fig. S2-S5, and ref. 28), suggesting that a degree of cooperativity may exist.

Our xanthine-based photoaffinity probes were designed to have the closest possible structural similarity to their parent compound, Pico145. The observation that the photoaffinity probes retain potency against all tested homo- and concatemeric TRPC1/4/5 channels implies that they can be used to assess cellular engagement of TRPC1, TRPC4 and TRPC5 in various tetramers. Considering its potency against TRPC4-C1 and TRPC5-C1 (Table 1), Pico145-DAAlk2 7 may be the most suitable photoaffinity probe for heteromeric or concatemeric TRPC1/4/5 channels. In addition, the competition PAL protocol may allow the assessment of target engagement of other TRPC1/4/5 modulators as well, and thereby support studies into their modes-of-action.

Pico145 and HC-070 are the most potent TRPC1/4/5 inhibitors reported to date, and in vitro screening of these compounds against hundreds of other proteins (ion channels, receptors, transporters and enzymes) suggested exquisite selectivity. ${ }^{23,25}$ Limited aqueous solubility and high plasma protein binding (PPB) have been highlighted as potential draw-backs of xanthine-based TRPC1/4/5 modulators, motivating the development of pyridazonebased TRPC4/5/inhibitors such as GFB-8438. ${ }^{53}$ Although GFB-8438 has lower potency against TRPC4/5 channels (TRPC4:C4 IC $50=$ $0.29 \mu \mathrm{M}$; TRPC5:C5 $\mathrm{IC}_{50}=0.18-0.28 \mu \mathrm{M}$ ), it has substantially higher solubility and lower PPB than Pico $145,{ }^{53}$ consistent with our calculations (ESI, $\dagger$ Table S1). However, the xanthines Pico145 and HC-070 have been used successfully for the modulation of TRPC1/4/5 channels in tissues and animals by multiple academic and industrial groups,${ }^{21,25-27}$ suggesting that the lower solubility and higher PPB of these xanthines may be off-set by their substantially higher potency. In addition, all four of our xanthine-based photoaffinity probes (and especially Pico145-DAAlk 5 lacking the non-essential 4-chlorobenzyl substituent on $N-7$ ) are predicted to have higher aqueous solubility than Pico145 (ESI, $\dagger$ Table S1), suggesting that further modification to the xanthines could address potential solubility issues if needed. Photoaffinity labelling with excess Pico145-DAAlk 5 in HEK T-REx cells suggested modification of multiple proteins (ESI, $\dagger$ Fig. S7), which is consistent with the hydrophobic nature and of Pico145-DAAlk 5 (favouring non-specific interactions with proteins) and the indiscriminate nature of diazirine-based photoaffinity labelling. In future studies, the use of our photoaffinity probes in comparative mass spectrometry-based chemical proteomics workflows (for example using Pico145 as a competitor) may enable the unbiased profiling of true targets and off-targets of xanthine-based TRPC1/4/5 modulators in cells and tissues.

Our PAL protocol provides the first method for the quantitative assessment of ligand engagement by TRPC5 channels, a crucial step in the drug discovery process. Because xanthines bind to a conserved, key modulatory TRPC1/4/5 channel binding site, competition PAL with xanthine-based photoaffinity probes may underpin the development of new TRPC1/4/5 modulators as chemical probes and drug candidates for a wide range of diseases including those of the CNS, kidney, heart, and cardiovascular system.

\section{Methods}

\section{Abbreviations and definitions}

TRPC, Transient Receptor Potential Canonical; EA, (-)-englerin A; S1P, sphingosine-1-phosphate; HEK T-REx cells; human embryonic kidney 293 cells stably expressing the tetracycline repressor protein, allowing tetracycline-inducible recombinant protein over-expression. TRPC1, TRPC4 and TRPC5 denote the different proteins or channels incorporating them; TRPC1/4/5 denotes channels composed of TRPC1, TRPC4 and/or TRPC5 (homo- or heteromeric; any ratio); TRPC4:C4 and TRPC5:C5 denote specific homomeric channels; TRPC1:C4 and TRPC1:C5 denote heteromeric channels formed by TRPC1 and either TRPC4 or TRPC5 (any ratio). TRPC4-C1 and TRPC5-C1 denote (channels composed of) recombinant, concatemeric proteins (fusions of TRPC1 at the C-terminus of either TRPC4 or TRPC5 through a short linker); ${ }^{18,23,28,54}\left[\mathrm{Ca}^{2+}\right]_{\mathrm{i}}$, intracellular concentration of $\mathrm{Ca}^{2+}$.

\section{Synthesis and characterization}

Full synthetic procedures and characterisation data are available in the ESI, $\dagger$ Methods.

\section{Cell culture and expression systems}

HEK T-REx cells expressing inducible TRPC5-SYFP2, TRPC4SYFP2, TRPC5-C1 and TRPC4-C1 have been described previously. ${ }^{18,23,28,54}$ Cells were maintained in Dulbecco's Modified Eagle Medium (DMEM), supplemented with foetal bovine serum (FBS; 10\%), penicillin-streptomycin (100 units per $\left.\mathrm{ml} ; 100 \mu \mathrm{g} \mathrm{ml}^{-1}\right)$ and blasticidin $\left(10 \mu \mathrm{g} \mathrm{ml}^{-1}\right)$ to maintain expression of the tetracycline repressor, and zeocin $\left(400 \mu \mathrm{g} \mathrm{ml}^{-1}\right)$ to maintain incorporation of the relevant TRPC plasmid. Cells were kept in a humidified incubator at $37{ }^{\circ} \mathrm{C}$ at $5 \% \mathrm{CO}_{2}$. Expression of TRPC protein was induced by the addition of $1 \mu \mathrm{g} \mathrm{m} \mathrm{m}^{-1}$ tetracycline (Merck, Gillingham, Dorset, UK) to the culture medium $24 \mathrm{~h}$ before experiments. 


\section{Intracellular $\mathrm{Ca}^{2+}$ measurements}

$\left[\mathrm{Ca}^{2+}\right]_{\mathrm{i}}$ recordings were carried out using the ratiometric $\mathrm{Ca}^{2+}$ dye Fura-2. $24 \mathrm{~h}$ before experiments, HEK T-REx cells stably transfected with relevant TRPC plasmids were plated onto black, clear-bottom, poly-D-lysine coated 96-well plates at 50000 cells per well. Cells were induced with $1 \mu \mathrm{g} \mathrm{ml}^{-1}$ tetracycline at this point. To load cells with the Fura-2 dye, media was removed and cells were incubated with standard bath solution (SBS) containing $2 \mu \mathrm{M}$ Fura-2 acetoxymethyl ester (Fura-2 AM; ThermoFisher Scientific, Waltham, MA, USA) and $0.01 \%$ pluronic acid (Merck) for $1 \mathrm{~h}$ at $37{ }^{\circ} \mathrm{C}$. SBS contained (in $\mathrm{mM}$ ): $\mathrm{NaCl} 135, \mathrm{KCl} 5$, glucose 8, HEPES 10, $\mathrm{CaCl}_{2} 1.5$ and $\mathrm{MgCl}_{2}$ 1.2. After this incubation, cells were washed with fresh SBS and incubated at room temperature for a further $30 \mathrm{~min}$. Immediately prior to the experiment, SBS was removed, and recording buffer (SBS $+0.01 \%$ pluronic acid $+0.01 \%$ DMSO) was added to cells. When assaying cells in inhibition mode, cells were washed briefly, twice, with SBS after Fura-2 AM incubation, and then incubated in recording buffer with inhibitors or DMSO for $30 \mathrm{~min}$ at $\mathrm{rt}$. $\left[\mathrm{Ca}^{2+}\right]_{\mathrm{i}}$ was measured in the FlexStation3 (Molecular Devices, Wokingham, UK), by alternating excitation at $340 \mathrm{~nm}$ and $380 \mathrm{~nm}$, with an emission at $510 \mathrm{~nm}$. Measurements were performed at $\mathrm{rt}$ for $5 \mathrm{~min}$ at $5 \mathrm{~s}$ intervals. Compounds used or tested as TRPC channel activators were dissolved at $2 \times$ final concentration in compound buffer (SBS $+0.01 \%$ pluronic acid) and added to cells after recording for $60 \mathrm{~s}$. When testing compounds as inhibitors, inhibitors or DMSO were added to the compound buffer at the same concentration as in the recording buffer.

\section{Docking studies}

The region of the TRPC5:C5 tetrameric structure (PDB: 6YSN) assigned for docking studies was chosen based upon residues known to be crucial for Pico145 binding (Y524, L572, Q573, F576, W577, V579, F599, Y603 and V614). A $36 \AA$ clip of the TRPC5 structure around these residues was termed as the receptor for docking studies using Glide (Schrödinger Release 2020-1, Glide, Schrödinger, LLC, New York, NY, 2020). ${ }^{55}$ The TRPC5 pdb file was prepared using the Protein Preparation Wizard in the Maestro Graphical User Interface (GUI). This aimed to remove any steric clashes of amino acid side chains and optimise the position of hydrogen atoms to facilitate docking studies. The receptor grid was generated using Schrödinger software, allowing docking of ligands in a $36 \times$ $36 \times 36 \AA^{3}$ 'grid'. Photoaffinity probes 4-7 were imported into the Maestro GUI and prepared using the LigPrep module to produce an energy minimised 3D structure. Docking of photoaffinity probes was carried out using the Glide module of Schrödinger software using the XP mode with flexible ligand sampling and biased sampling of torsions for all predefined functional groups. Epik state penalties were added to the docking score. A maximum of 10 poses were requested in the output file for each ligand and post-docking minimisation was carried out. Docking of probes into the defined Pico145 binding site of TRPC5:C5 produced three poses for each compound with similar predicted binding energies. These poses were almost identical with the lowest energy pose making key hydrogen bonding interactions between the flexible hydroxypropyl groups and the side chain of Q573. Critical $\pi$-stacking interactions were seen between the xanthine cores and F576, and between the $C$-8 aryl substituents and Y524. To modify the prepared Pico145 structure to give Pico145-DA (in ESI, $\dagger$ Fig. S6), the build tool in the Maestro GUI was used to swap the $\mathrm{OCF}_{3}$ for a diazirine- $\mathrm{CF}_{3}$ moiety and this section of the ligand was minimised, leaving stationary the remaining core of the ligand and amino acids in proximity to the group. After minimisation, the proximity of the diazirine moiety to nearby residues was measured.

\section{Calculation of predicted solubility}

The predicted $\log S$ and $\log P$ values for energy-minimised versions of Pico145, AM237, photoaffinity probes 4-7 and GFB-8438 (ESI, $\dagger$ Table S1) were calculated using QikProp (Schrödinger Release 2020-1, Glide, Schrödinger, LLC, New York, NY, 2020).

\section{Photoaffinity labelling protocol}

Reagents. TAMRA biotin azide (ESI, $\dagger$ Fig. S35) was obtained from Click Chemistry Tools (Scottsdale, AZ, USA), made up to $10 \mathrm{mM}$ in dry DMSO and stored in aliquots at $-20{ }^{\circ} \mathrm{C}$. $\mathrm{CuSO}_{4}$ (Acros) and tris(2-carboxyethyl)phosphine (TCEP; Acros) were made up in degassed $\mathrm{H}_{2} \mathrm{O}$ to $50 \mathrm{mM}$ on the day of use and stored on ice. Tris[(1-benzyl-1H-1,2,3-triazol-4-yl)methyl]amine (TBTA; Alfa Aesar) was made up in dry DMSO to $80 \mathrm{mM}$ on the day of use.

Photoaffinity labelling (PAL) with Pico145-DAAlk 5. HEK T-REx cells expressing inducible TRPC5-SYFP2 were plated onto poly-D-lysine coated $10 \mathrm{~cm}$ dishes at $7 \times 10^{6}$ cells per dish, and left to adhere. After 24 hours, medium was removed and replaced with fresh medium containing $1 \mu \mathrm{g} \mathrm{ml} \mathrm{m}^{-1}$ tetracycline to induce expression of TRPC5-SYFP2. For control cells not expressing TRPC5-SYFP2, medium was changed to fresh medium without tetracycline. Cells were used for photoaffinity labelling $24 \mathrm{~h}$ after induction. To treat cells for photoaffinity labelling, medium was removed and cells were washed once in PBS + $1 \mathrm{mM}$ $\mathrm{CaCl}_{2}$. SBS containing photoaffinity probe (Pico145-DAAlk, 5) was then added to the cells, and cells were incubated at $37{ }^{\circ} \mathrm{C}$ for $30 \mathrm{~min}$. For competition experiments, cells were treated in SBS containing either DMSO or Pico145 at $37{ }^{\circ} \mathrm{C}$ for $30 \mathrm{~min}$ before being replaced with SBS containing photoaffinity probe as well as DMSO or inhibitor. Cells treated without photoaffinity probe were treated with DMSO to the same concentration. Following incubation, lids were removed from the dishes, and cells irradiated at $365 \mathrm{~nm}$ for $25 \mathrm{~min}$ using a UV crosslinker (Analytik Jena AG, Jena, Germany). SBS was then removed, and cells were washed $3 \times$ with $\mathrm{PBS}+1 \mathrm{mM} \mathrm{Ca}{ }^{2+}$, followed by addition of $400 \mu \mathrm{l}$ RIPA buffer containing protease inhibitor cocktail (EDTA-free; ThermoFisher Scientific). RIPA buffer contained: Tris/HCl (10 mM, pH 7.5), NaCl (150 mM), EDTA (0.5 mM), SDS $(0.1 \% \mathrm{w} / \mathrm{v})$, Triton X-100 (1\% v/v), deoxycholate $(1 \% \mathrm{w} / \mathrm{v})$. Cells were scraped into $1.5 \mathrm{ml}$ Eppendorf tubes, and rotated for $1 \mathrm{~h}$ at $4{ }^{\circ} \mathrm{C}$. Lysates were then cleared by centrifugation at $10000 \mathrm{~g}$ for 
$10 \mathrm{~min}$ at $4{ }^{\circ} \mathrm{C}$ and transferred to fresh tubes. At this stage, input samples were taken by removal of $20 \mu \mathrm{l}$ of lysate. To these input samples, $4 \times$ loading buffer (BioRad, Watford, UK) $+10 \%$ beta-mercaptoethanol was added, and samples were boiled at $95{ }^{\circ} \mathrm{C}$ for $10 \mathrm{~min}$, then stored at $-20{ }^{\circ} \mathrm{C}$. Protein concentrations for the remaining lysates were quantified using bicinchoninic acid (ThermoFisher Scientific), followed by immunoprecipitation of TRPC5-SYFP2. TRPC5-SYFP2 was immunoprecipitated using GFP-Trap agarose (Chromotek, Planegg, Germany) according to manufacturer's instructions. Samples were adjusted to $2.5 \mathrm{mg}$ protein and equal volume in dilution buffer $(10 \mathrm{mM}$ Tris/HCl pH 7.5, $150 \mathrm{mM} \mathrm{NaCl}, 0.5 \mathrm{mM}$ EDTA), before addition of $20 \mu$ washed bead slurry per sample. Immunoprecipitations were incubated with end-over-end mixing at $4{ }^{\circ} \mathrm{C}$ for $3 \mathrm{~h}$, followed by 3 washes in wash buffer $(10 \mathrm{mM}$ Tris/ $\mathrm{HCl} \mathrm{pH} 7.5,150 \mathrm{mM}$ $\mathrm{NaCl}$, $0.5 \mathrm{mM}$ EDTA), to remove non-specific binders. Following removal of wash buffer, immunoprecipitated proteins were resuspended in $74 \mu \mathrm{l}$ PBS $+1 \%$ SDS. Proteins were then tagged with biotin by on-bead copper-catalysed azide-alkyne cycloaddition (CuAAC). Reagents (TAMRA biotin azide $100 \mu \mathrm{M}$, $\mathrm{CuSO}_{4} 1 \mathrm{mM}$, TCEP $1 \mathrm{mM}$, TBTA $800 \mu \mathrm{M}$ ) were added to immunoprecipitated proteins and reactions incubated at $37^{\circ} \mathrm{C}$ for $1 \mathrm{~h}$ with shaking at $250 \mathrm{rpm}$. Reactions were quenched by addition of $10 \mathrm{mM}$ EDTA, and precipitations washed $3 \times$ in wash buffer (3000 g, $5 \mathrm{~min}$ ). After removal of the last wash, GFP-Trap beads were resuspended in $45 \mu \mathrm{l} 2 \%$ SDS in PBS and $25 \mu \mathrm{l} 4 \times$ loading buffer containing $10 \%(\mathrm{v} / \mathrm{v})$ betamercaptoethanol, and boiled for $95{ }^{\circ} \mathrm{C}$ for $10 \mathrm{~min}$ to dissociate protein from GFP-Trap beads, and stored at $-20{ }^{\circ} \mathrm{C}$ prior to SDS-PAGE.

Photoaffinity labelling (PAL) with Pico145-DAAlk2. Experiments using the Pico145-DAAlk2 probe were performed using a modified method of that described for Pico145-DAAlk. HEK T-REx cells expressing inducible TRPC5-SYFP2 were plated onto poly-D-lysine coated 6 well plates at $1 \times 10^{6}$ cells per well, and left to adhere for $24 \mathrm{~h}$, before induction of protein expression as described above. Cells were treated with Pico145-DAAlk2 7 as described above, but were irradiated at $365 \mathrm{~nm}$ for 1 minute with our home-built LED photocrosslinker (see ESI, $\dagger$ Methods for details). After lysis in $100 \mu$ l RIPA buffer, samples were quantified and adjusted the lowest concentration $(0.5 \mathrm{mg}$ protein and equal volume) and immunoprecipitated overnight at $4{ }^{\circ} \mathrm{C}$. CuAAC reactions were then carried out as described for Pico145-DAAlk.

SDS-PAGE and western blotting. Prior to SDS-PAGE, PAL samples were centrifuged $(5000 \mathrm{~g}, 1 \mathrm{~min})$ to pellet GFP-Trap beads. PAL and input samples were separated by SDS-PAGE using 4-15\% pre-cast gels (BioRad), before being transferred to PVDF membrane (Merck Millipore, Burlington, MA, USA) using wet transfer (350 mA, 1 hour). Following transfer, membranes were blocked in either 5\% BSA (PAL samples) or 5\% skimmed milk (input) in PBS-T for $1 \mathrm{~h}$ at rt. Membranes were then probed with Streptavidin-HRP (PAL samples, 1 : 5000; ThermoFisher Scientific) or anti-GFP (input, 1: 8000; Abcam, Cambridge, UK in blocking buffer, overnight at $4{ }^{\circ} \mathrm{C}$ ). For input samples membranes were washed in PBS-T $(6 \times 5 \mathrm{~min}, \mathrm{rt})$ before incubation in secondary antibody (anti-mouse HRP, 1:5000;
ThermoFisher Scientific) for $1 \mathrm{~h}$ at $\mathrm{rt}$, and further washing in PBS-T. For PAL samples, membranes were washed in PBS-T $(6 \times 5 \mathrm{~min}, \mathrm{rt})$. Following washing, blots were imaged using ECL (ThermoFisher Scientific) and G-box (Syngene, Bangalore, India). To reprobe PAL membranes, membranes were stripped in Restore Stripping buffer (20 min, rt; ThermoFisher Scientific), washed in PBS $(3 \times 5 \mathrm{~min}, \mathrm{rt})$, and blocked in 5\% milk in PBS-T. Membranes were then incubated with anti-GFP $(1: 8000,2 \mathrm{~h}, \mathrm{rt})$, washed with PBS-T, incubated with anti-mouse $\operatorname{HRP}(1: 5000,1 \mathrm{~h}$, rt; ThermoFisher Scientific), washed with PBS-T and imaged using ECL and G-box. Blots were quantified using ImageJ.

Data analysis. Data from $\left[\mathrm{Ca}^{2+}\right]_{\mathrm{i}}$ measurements were analysed using Microsoft Excel and GraphPad Prism, and presented as mean \pm SEM. Statistical tests were performed as described in figure legends, with significance decided at $p<0.05 .\left[\mathrm{Ca}^{2+}\right]_{\mathrm{i}}$ assays were performed as 3 independent replicates, with each replicate containing 6 technical replicates (6 wells of a 96-well plate; i.e. $n / N=3 / 18)$, unless indicated otherwise. Responses were calculated at the time points indicated, compared to the baseline at 0-55 s. These results were used to construct concentrationresponse curves and calculate $\mathrm{EC}_{50}$ and $\mathrm{IC}_{50}$ values in GraphPad Prism. Photoaffinity labelling experiments were performed as 3 independent experiments $(n=3)$, unless described otherwise. Western blot densitometry analysis was performed using ImageJ, comparing \% biotin/GFP compared to a relevant control sample as described.

Data availability. The authors declare that the data supporting the findings of this study are available within the article and ESI, $\dagger$ or from the corresponding author upon reasonable request.

\section{Author contributions}

AM, IBP and RSB designed and synthesised photoaffinity probes. $\mathrm{CCB}, \mathrm{AM}$ and IBP carried out calcium measurements and photoaffinity labelling experiments. IBP and KJS performed docking studies. KJS performed calculations of compound solubilities. CCB, AM, IBP, KJS, ECB and RSB analysed data. NK developed the LED-based photocrosslinker. MPB, DJB, SPM, MHW and SLW advised on experimental design and data interpretation, and generated research funds. RSB conceived and led the project, and generated research funds. CCB, IBP, ECB, NK and RSB made figures. CCB, IBP, KJS and RSB wrote the manuscript. All authors commented on the manuscript.

\section{Conflicts of interest}

DJB is an inventor on the following patent applications: 1) PCT/GB2018/050369. TRPC ion channel inhibitors for use in therapy. Filing date: $9^{\text {th }}$ February 2018. Inventors: David J Beech, Richard J Foster, Sin Ying Cheung and Baptiste M Rode; 2) $62 / 529,063$. Englerin derivatives for treatment of cancer. Filing date: $6^{\text {th }}$ July 2017. Inventors: John A Beutler, Antonio Echavarren, William Chain, David Beech, Zhenhua Wu, Jean-Simon Suppo, Fernando Bravo and Hussein Rubaiy. 


\section{Acknowledgements}

This work was supported by the BBSRC (BB/P020208/1), the BHF (PG/19/2/34084), a BBSRC/AstraZeneca iCASE PhD studentship (BB/L015676/1) to AM, and a Wellcome Trust PhD studentship $(102174 / \mathrm{B} / 13 / \mathrm{Z})$ to IBP and a Wellcome Trust Award to DJB (10044/Z/15/Z). We thank Lucia Pedicini, Katarina Miteva and Lynn McKeown for discussions, Martin Walko for donation of a sample of diazirine building block 22, and Devon Legge for donation of a sample of TAMRA biotin azide used in pilot photoaffinity labelling experiments. Docking studies in this work were undertaken on ARC3, part of the High Performance Computing facilities at the University of Leeds, UK. Fig. 4 and the TOC figure were produced using biorender.com.

\section{References}

1 J. Abramowitz and L. Birnbaumer, Physiology and pathophysiology of canonical transient receptor potential channels, FASEB J., 2009, 23, 297-328.

2 R. S. Bon and D. J. Beech, In pursuit of small molecule chemistry for calcium-permeable non-selective TRPC channels - mirage or pot of gold?, Br. J. Pharmacol., 2013, 170, 459-474.

3 K. Venkatachalam and C. Montell, TRP Channels, Annu. Rev. Biochem., 2007, 76, 387-417.

4 C. Montell, The TRP superfamily of cation channels, Sci. STKE, 2005, re3.

5 T. Voets, K. Talavera, G. Owsianik and B. Nilius, Sensing with TRP channels, Nat. Chem. Biol., 2005, 1, 85-92.

6 C. Montell, et al., A Unified nomenclature for the superfamily of TRP cation channels, Mol. Cell, 2002, 9, 229-231.

$7 \mathrm{X}$. Zhu, et al., trp, a novel mammalian gene family essential for agonist-activated capacitative $\mathrm{Ca}^{2+}$ entry, Cell, 1996, 85, 661-671.

8 P. D. Wes, et al., TRPC1, a human homolog of a Drosophila store-operated channel, Proc. Natl. Acad. Sci. U. S. A., 1995, 92, 9652-9656.

9 D. E. Clapham, L. W. Runnels and C. Strübing, The trp ion channel family, Nat. Rev. Neurosci., 2001, 2, 387-396.

10 B. Vannier, et al., Mouse trp2, the homologue of the human trpc2 pseudogene, encodes mTrp2, a store depletionactivated capacitative $\mathrm{Ca}^{2+}$ entry channel, Proc. Natl. Acad. Sci. U. S. A., 1999, 96, 2060-2064.

11 A. Minard, et al., Remarkable progress with small-molecule modulation of TRPC1/4/5 channels: implications for understanding the channels in health and disease, Cells, 2018, 7,52 .

12 H. Wang, et al., TRPC channels: structure, function, regulation and recent advances in small molecular probes, Pharmacol. Ther., 2020, 107497.

13 S. Suresh Babu, et al., Mechanism of Stretch-Induced Activation of the Mechanotransducer Zyxin in Vascular Cells, Sci. Signaling, 2012, 5, ra91.

14 P. Sukumar, et al., Constitutively active TRPC channels of adipocytes confer a mechanism for sensing dietary fatty acids and regulating adiponectin, Circ. Res., 2012, 111, 191-200.

15 M. Gautier, et al., New insights into pharmacological tools to TR(i)P cancer up, Br. J. Pharmacol., 2014, 171, 2582-2592.

16 S. Sharma and C. R. Hopkins, A review of transient receptor potential channel (TRPC) modulators and diseases, J. Med. Chem., 2019, 62, 7589-7602.

17 Y. Akbulut, et al., (-)-Englerin A is a potent and selective activator of TRPC4 and TRPC5 calcium channels, Angew. Chem., Int. Ed., 2015, 54, 3787-3791.

18 M. J. Ludlow, et al., (-)-Englerin A-evoked cytotoxicity is mediated by $\mathrm{Na}^{+}$influx and counteracted by $\mathrm{Na}^{+} / \mathrm{K}^{+}-$ ATPase, J. Biol. Chem., 2017, 292, 723-731.

19 C. Carson, et al., Englerin A agonizes the TRPC4/C5 cation channels to inhibit tumor cell line proliferation, PLoS One, 2015, 1, 1-21.

$20 \mathrm{X}$. Wang, et al., TRPC5 does not cause or aggravate glomerular disease, J. Am. Soc. Nephrol., 2018, 29, 409-415.

21 S. Y. Cheung, et al., TRPC4/TRPC5 channels mediate adverse reaction to the cancer cell cytotoxic agent (-)-Englerin A, Oncotarget, 2018, 9, 29634-29643.

22 B. L. Chenard and R. J. Gallaschun, Substituted xanthines and methods of use thereof, International patent WO/2014/ 143799, 2014.

23 H. N. Rubaiy, et al., Picomolar, selective, and subtypespecific small-molecule inhibition of TRPC1/4/5 channels, J. Biol. Chem., 2017, 292, 8158-8173.

24 H. N. Rubaiy, M. J. Ludlow, R. S. Bon and D. J. Beech, Pico145 - powerful new tool for TRPC1/4/5 channels, Channels, 2017, 11, 362-364.

25 S. Just, et al., Treatment with HC-070, a potent inhibitor of TRPC4 and TRPC5, leads to anxiolytic and antidepressant effects in mice, PLoS One, 2018, 1, 1-32.

26 S. Lepannetier, et al., Activation of TRPC1 channel by metabotropic glutamate receptor mGluR5 modulates synaptic plasticity and spatial working memory, Front. Cell. Neurosci., 2018, 12, 318.

27 Y. Yu, et al., Development of a carbon-11 PET radiotracer for imaging TRPC5 in the brain, Org. Biomol. Chem., 2019, 17, 5586-5594.

28 A. Minard, et al., Potent, selective, and subunit-dependent activation of TRPC5 channels by a xanthine derivative, Br. J. Pharmacol., 2019, 176, 3924-3938.

29 D. J. Wright, et al., Cryo-EM structures of human TRPC5 reveal interaction of a xanthine-based TRPC1/4/5 inhibitor with a conserved lipid binding site, bioRxiv, 2020, DOI: 10.1101/2020.04.17.047456.

30 K. Song, et al., Structural basis for human TRPC5 channel inhibition by two distinct inhibitors, bioRxiv, 2020, DOI: 10.1101/2020.04.21.052910.

31 G. M. Simon, M. J. Niphakis and B. F. Cravatt, Determining target engagement in living systems, Nat. Chem. Biol., 2013, 9, 200-205.

32 M. Schürmann, P. Janning, S. Ziegler and H. Waldmann, Small-molecule target engagement in cells, Cell Chem. Biol., 2016, 23, 435-441. 
33 M. Schenone, V. Dančík, B. K. Wagner and P. A. Clemons, Target identification and mechanism of action in chemical biology and drug discovery, Nat. Chem. Biol., 2013, 9, 232-240.

34 M. E. Bunnage, A. M. Gilbert, L. H. Jones and E. C. Hett, Know your target, know your molecule, Nat. Chem. Biol., 2015, 11, 368-372.

35 V. Chowdhry and F. H. Westheimer, Photoaffinity labeling of biological systems, Annu. Rev. Biochem., 1979, 48, 293-325.

36 E. Smith and I. Collins, Photoaffinity labeling in target- and binding-site identification, Future Med. Chem., 2015, 7, 159-183.

37 G. W. Preston and A. J. Wilson, Photo-induced covalent cross-linking for the analysis of biomolecular interactions, Chem. Soc. Rev., 2013, 42, 3289-3301.

38 K. J. Gregory, et al., Clickable Photoaffinity ligands for metabotropic glutamate receptor 5 based on select acetylenic negative allosteric modulators, ACS Chem. Biol., 2016, 11, 1870-1879.

39 S. Kiyonaka, et al., Selective and direct inhibition of TRPC3 channels underlies biological activities of a pyrazole compound, Proc. Natl. Acad. Sci. U. S. A., 2009, 106, 5400-5405.

40 G. M. S. Yip, et al., A propofol binding site on mammalian GABAA receptors identified by photolabeling, Nat. Chem. Biol., 2013, 9, 715-720.

41 K. A. Woll, et al., Photoaffinity ligand for the inhalational anesthetic sevoflurane allows mechanistic insight into potassium channel modulation, ACS Chem. Biol., 2017, 12, 1353-1362.

42 M. Soethoudt, et al., Selective photoaffinity probe that enables assessment of cannabinoid $\mathrm{CB}_{2}$ receptor expression and ligand engagement in human cells, J. Am. Chem. Soc., 2018, 140, 6067-6075.

43 J. Park, M. Koh, J. Y. Koo, S. Lee and S. B. Park, Investigation of specific binding proteins to photoaffinity linkers for efficient deconvolution of target protein, ACS Chem. Biol., 2016, 11, 44-52.

44 T. Kambe, B. E. Correia, M. J. Niphakis and B. F. Cravatt, Mapping the protein interaction landscape for fully functionalized small-molecule probes in human cells, J. Am. Chem. Soc., 2014, 136, 10777-10782.

45 R. A. G. Smith and J. R. Knowles, Aryldiazirines. Potential reagents for photolabeling of biological receptor sites, J. Am. Chem. Soc., 1973, 95, 5072-5073.

46 L. Dubinsky, B. P. Krom and M. M. Meijler, Diazirine based photoaffinity labeling, Bioorg. Med. Chem., 2012, 20, 554-570.

47 J. R. Hill and A. A. B. Robertson, Fishing for drug targets: a focus on diazirine photoaffinity probe synthesis, J. Med. Chem., 2018, 61, 6945-6963.

48 J. Das, Aliphatic diazirines as photoaffinity probes for proteins: recent developments, Chem. Rev., 2011, 111, 4405-4417.

49 V. V. Rostovtsev, L. G. Green, V. V. Fokin and K. B. Sharpless, A stepwise Huisgen cycloaddition process: copper(I)-catalyzed regioselective "ligation" of azides and terminal alkynes, Angew. Chem., Int. Ed., 2002, 41, 2596-2599.

50 J. Brunner, H. Senn and F. M. Richards, 3-Trifluoromethyl3-phenyldiazirine. A new carbene generating group for photolabeling reagents, J. Biol. Chem., 1980, 255, 3313-3318.

51 J. Duan, et al., Cryo-EM structure of TRPC5 at 2.8-Å resolution reveals unique and conserved structural elements essential for channel function, Sci. Adv., 2019, 5, eaaw7935.

52 G. W. Preston, S. E. Radford, A. E. Ashcroft and A. J. Wilson, Covalent cross-linking within supramolecular peptide structures, Anal. Chem., 2012, 84, 6790-6797.

$53 \mathrm{M}$. Yu, et al., Discovery of a potent and selective TRPC5 inhibitor, efficacious in a focal segmental glomerulosclerosis model, ACS Med. Chem. Lett., 2019, 10, 1579-1585.

54 H. N. Rubaiy, et al., Tonantzitlolone is a nanomolar potency activator of transient receptor potential canonical 1/4/5 channels, Br. J. Pharmacol., 2018, 175, 3361-3368.

55 R. A. Friesner, et al., Extra precision glide: docking and scoring incorporating a model of hydrophobic enclosure for protein-ligand complexes, J. Med. Chem., 2006, 49, 6177-6196. 